\title{
A remark on the Upper Bounds of the Moduli of the Roots of Algebraic Equations
}

\author{
Dragomir M. Simeunović
}

Abstract. In this paper we obtain one upper bound of the moduli of the roots of the algebraic equations.

The bounds of the moduli of the roots of algebraic equations were researched by many authors (see e.g. $[1,2,3,4]$ ).

For the algebraic equation

$$
z^{n}+a_{1} z^{n-1}+a_{2} z^{n-2}+\cdots+a_{n-1} z+a_{n}=0
$$

let

$$
\left|a_{k}\right|=A_{k}, \quad k=1,2, \ldots, n .
$$

In this paper, for the equation (1) the following theorem is proved.

Theorem. Let $C_{k}, k=1,2, \ldots, n$ be positive parameters for which

(3) $\left(C_{1}+A_{1}\right)^{2}-4 C_{2} \geq 0 ;\left(2 C_{k}+A_{k}\right)^{2}-4 C_{k-1} C_{k+1} \geq 0 ; k=2,3, \ldots, n$.

\section{Then}

(4)

$$
R=\max \left(\begin{array}{l}
\frac{C_{1}+A_{1}+\sqrt{\left(C_{1}+A_{1}\right)^{2}-4 C_{2}}}{2} ; \\
\frac{2 C_{k}+A_{k}+\sqrt{\left(2 C_{k}+A_{k}\right)^{2}-4 C_{k-1} C_{k+1}}}{2 C_{k-1}}
\end{array}, k=2,3, \ldots, n-1 ;\right)
$$

is one upper bound for the moduli of the roots of the equation (1).

Proof. Let $z=r e^{\theta i}(0 \leq \theta<2 \pi)$ be the root of the equation (1), where

$$
|z|=r .
$$

Taking into account (2) and (5), from (1) we obtain the inequality

$$
r^{n} \leq A_{1} r^{n-1}+A_{2} r^{n-2}+\cdots+A_{n-1} r+A_{n} .
$$

2000 Mathematics Subject Classification. Primary: 12D10.

Key words and phrases. Roots of algebraic equations, upper bounds for roots moduli. 
Let $R$ be a positive number for which

$$
R^{n} \geq A_{1} R^{n-1}+A_{2} R^{n-2}+\cdots+A_{n-1} R+A_{n} .
$$

Then, according to Cauchy's theorem (see [1], p. 122) for the moduli of the roots of the equation (1) we have the following relation

$$
r \leq R
$$

Let the parameters $C_{k}>0, k=1,2, \ldots, n$ satisfy the following inequalities

(9)

$$
\left\{\begin{array}{l}
R^{n}-C_{1} R^{n-1}+C_{2} R^{n-2} \geq A_{1} R^{n-1} \\
C_{1} R^{n-1}-2 C_{2} R^{n-2}+C_{3} R^{n-3} \geq A_{2} R^{n-2} \\
\quad \vdots \\
C_{n-2} R^{2}-2 C_{n-1} R+C_{n} \geq A_{n-1} R \\
C_{n-1} R-C_{n} \geq A_{n} .
\end{array}\right.
$$

The sum of all inequalities in (9) gives the inequality (7).

The inequalities (9) are satisfied for

$$
\begin{aligned}
& R \geq \frac{C_{1}+A_{1}+\sqrt{\left(C_{1}+A_{1}\right)^{2}-4 C_{2}}}{2} \\
& R \geq \frac{2 C_{k}+A_{k}+\sqrt{\left(2 C_{k}+A_{k}\right)^{2}-4 C_{k-1} C_{k+1}}}{2 C_{k-1}}, k=2,3, \ldots, n-1, \\
& R \geq \frac{C_{n}+A_{n}}{C_{n-1}} .
\end{aligned}
$$

The relations (10), (11) and (12) are satisfied for $R$ represented by (4), which completes the proof of the theorem, from whence follows the relation (8).

By giving the parameters $C_{k}, k=1,2, \ldots, n$ different positive values, we obtain from (4) the particular results.

For

$$
C_{k}=k+\sqrt{5}, \quad k=1,2, \ldots, n,
$$

and in case that

$$
A_{k} \leq k, \quad k=1,2, \ldots, n
$$

having in mind that

$$
3 k+k \sqrt{5}+2+2 \sqrt{5}=(k-1+\sqrt{5})(3+\sqrt{5}),
$$

we obtain the Montel's result from (4)

$$
R=\frac{3+\sqrt{5}}{2} .
$$

Remark. The result (15) also holds if

$$
A_{k} \leq k, \quad k=1,2, \ldots, n-1,
$$


and

$$
A_{n} \leq 1+\frac{(1+\sqrt{5}) n}{2} .
$$

In this paper the result (15) is obtained without using the infinite series.

\section{REFERENCES}

[1] M. Marden, Geometry of Polynomials, Amer. Math. Soc. Providence, R.I. 1966.

[2] S. Zervos, Aspects modernes de la localisation des zéros des polynômes d'une variable, Ann. Sci. École Norm. Sup., (3) 77(1960), 303-410.

[3] P. Montel, Sur quelques limites pour les modules des zéros des polynômes, Comment. Math. Helv., 7(1934-35), 178-200.

[4] D. Marković, Granice korena algebarskih jednačina, Srpska Kraljevska Akademija, Glas CLXXXI, prvi razred 90, A. Matematičke nauke, Beograd 1939, 117-130.

Dragomir M. Simeunović Mike Alasa 8

11000 BeLgRADE

SERBIA 